\title{
Corrupted Colonic Crypts Bordering Regenerating Mucosal Ulcers in Ulcerative Colitis
}

\author{
CARLOS A. RUBIO \\ Gastrointestinal and Liver Research Laboratory, Department of Pathology, \\ Karolinska Institute and University Hospital, Stockholm, Sweden
}

\begin{abstract}
Background/Aim: Histology in protracted ulcerative colitis (UC) discloses high numbers of chronic inflammatory cells and crypts with architectural distortions. In severe cases, ulcerations are frequently found. The histogenesis of colonic crypts with architectural distortions in UC remains elusive. A recent review of colectomy specimens from patients with UC revealed crypts surrounding mucosal ulcerations exhibiting severe architectural distortions. They were called corrupted colonic crypts, CCCs. Material and Methods: Archival hematoxylin and eosin $(H \& E)$-stained sections from three colectomies having several mucosal ulcers were selected for the study. The mucosa bordering mucosal ulcers was particularly scrutinized. Results: The review of 49 sections (mean=16.3, range $=14-20$ ) in the three colectomies revealed 60 ulcers ( mean=20, range $=13-27$ ). The following CCC phenotypes were found bordering mucosal ulcers: with asymmetric lateral fission $(n=11)$, with dual or three-foiled corrupted fission ( $n=19)$, with cystic dilatations $(n=3)$, L-shaped crypts $(n=7)$, T-inverted crypts $(n=6)$, shoe-shaped crypts $(n=3)$, horizontal crypts $(n=14)$, multi-lobate crypts $(n=2)$, and/or inter-connecting crypts $(n=5)$. Conclusion: The regeneration of ulcers in UC seems to proceed with neo-formation of corrupted crypts. In the same colectomies, none to occasional CCCs were found in large areas of the mucosa having severe chronic inflammation. Importantly, none of the occasional CCCs were found in other diseases of the colonic mucosa with chronic inflammation or in unspecific ulcers of
\end{abstract}

This article is freely accessible online.

Correspondence to: Dr. Carlos A. Rubio, Gastrointestinal and Liver Pathology Research Laboratory, Department of Pathology, Karolinska Institute and University Hospital, 17176, Stockholm, Sweden. Tel: +46 851774527, Fax: +46 851774524, e-mail: Carlos.Rubio@ki.se

Key Words: Colon, colectomy specimens, mucosal ulcers, ulcerative colitis crypts. the colon. Since neither chronic mucosal inflammation per se, nor unspecific ulcers of the colon are central for the formation of CCCs, it is suggested that crypt distortions of the non-ulcerated colonic mucosa in patients with UC might mirror formerly healed mucosal ulcerations.

The histologic examination of biopsies or surgical specimens from patients with protracted ulcerative colitis (UC) reveals high numbers of chronic inflammatory cells and distortion of the shape of the crypts. Granulocytes are found during the active phase of the disease and ulcerations are frequently found in severe cases.

Distortion of the shape of the crypts in UC has received various descriptive trivial names in the literature, such as alterations in crypt configuration (1), crypt distortions (2), crypt branching (3) and architectural aberrations (4), to name some. Nevertheless, the histogenesis of distorted crypts in UC has remained unexplored.

In previous work, we found non-dysplastic distorted colonic crypts of various shapes intercalated between normal crypts, in histologic sections from the colon of rats treated with different carcinogens $(5,6)$. Those distorted colonic crypts were called corrupted colon crypts (CCCs). More recently, while reviewing colectomy specimens from patients with severe UC we found similar distorted crypts surrounding mucosal ulcerations.

The purpose of this communication was to report and to illustrate the abnormal crypts evolving at the edge of mucosal ulcers in colectomy specimens from patients with UC.

\section{Materials and Methods}

Archival sections from three colectomies exhibiting a high number of mucosal ulcers were selected for the study. Surgery was performed because of severe UC refractory to clinical treatment. The histological sections were stained with hematoxylin and eosin (H\&E). The mucosa bordering mucosal ulcers was particularly scrutinized. Ulcers with re-epithelization were regarded those being partially or totally covered by a single-cell layer. As non-dysplastic CCCs bordering mucosal ulcers were regarded those portraying one or more alterations (see Table I). 

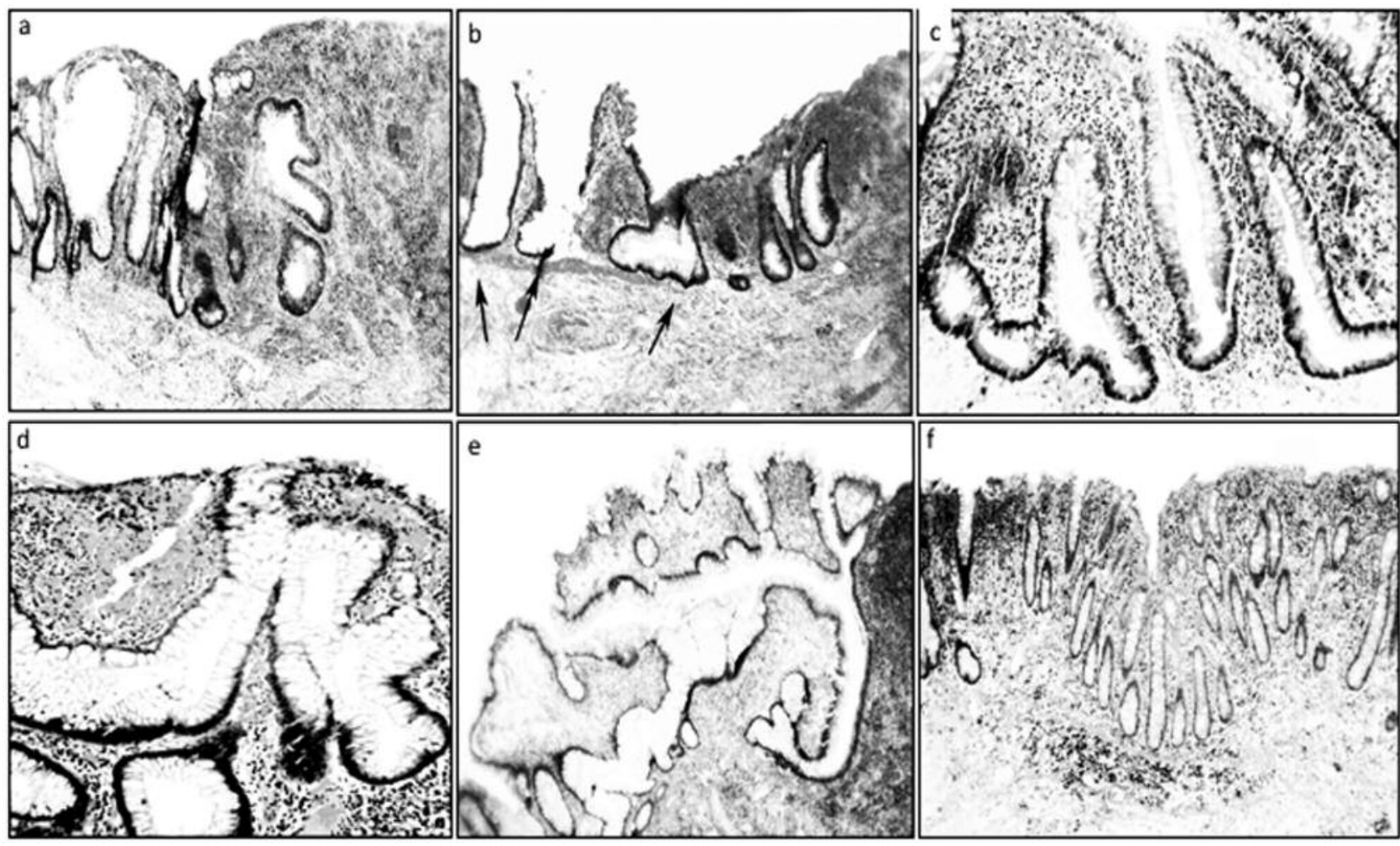

Figure 1. Regenerating ulcers in ulcerative colitis (UC). a: Corrupted cystic crypts with asymmetric fission (vertical arrows), and horizontal fission (horizontal arrow) $(H \& E, \times 4), b$ : Two hyperplastic, dilated crypts (left arrows) and one shoe-shaped crypt (central arrow). Note the absence of muscularis mucosa beyond the central arrow ( $H \& E$, magnification $\times 4), c$ : Crypts with asymmetric fission (upper crypt), one inverted T-shaped (left) and one L-shaped (right). (H\&E, magnification $\times 10)$, d: Multi-lobate crypts with asymetric fission (H\&E, magnification $\times 20)$, e: Crypt showing a central dilatation connected with five corrupted crypts, the one in the right ending in a three-foiled fission (H\&E, magnification $\times 4), f: F o r$ comparison, colonic mucosa with chronic inflammation in UC without ulcerations showing no corrupted crypts (H\&E, magnification $\times 4)$.

\section{Results}

A total of 49 filed H\&E-stained colonic sections (mean=16.3, range $=14-20$ ) were available for review. A total of 60 ulcers (mean $=20$, range 13-27) were found.

The results, condensed in Table I, show the frequency of the various CCC phenotypes bordering mucosal ulcers in the three specimens.

In addition, two of the 22 ulcers showed partial or total re-epithelization of the luminal aspect of the ulcer. Examples of some $\mathrm{CCC}$ phenotypes bordering ulcers in UC are shown in Figure 1.

\section{Discussion}

The results of this work indicate that the regeneration of ulcers in the colonic mucosa of patients with UC proceeds with neo-formation of crypts exhibiting corrupted shapes. Why the normal process of crypt regeneration in the colonic mucosa bordering UC ulcers is severely altered, remains
Table I. The number of corrupted colonic crypts (CCCs) found adjacent to colonic ulcers in colectomy specimens from patients with protracted ulcerative colitis (UC).

\begin{tabular}{lc}
\hline CCCs bordering ulcers in UC & No. CCCs* \\
\hline With asymmetric lateral fission & 11 \\
With dual or three-foiled fission & 19 \\
Cystic crypts & 3 \\
L-shaped crypts & 7 \\
T-inverted crypts & 6 \\
Shoe-shaped crypts & 3 \\
Horizontal crypts & 14 \\
Multi-lobate crypts & 2 \\
Inter-connecting crypts & 5 \\
\hline Total & 70
\end{tabular}

*In some cases, $>1$ phenotypes of CCC were found.

elusive. It is known that colonic crypts replicate by symmetric fission, beginning at the base of the crypts and proceeding upwards until two identical, individual crypts are 
created $(7,8)$. During crypt renewal, stem cells at the crypt bottom generate amplifying daughter cells that proliferate and differentiate while migrating upwards (8). APC normally down-regulates WNT signaling. The APC concentration is low at the crypt bottom and high at the top (the domain of differentiated cells). In contrast, WNT signaling is high at the bottom (where stem cells reside) and low at the top. Hence, WNT and APC gradients are important in crypt formation and regulation. Since both APC and WNT signaling components (e.g. survivin) are required for mitosis, this mechanism establishes a zone in the lower crypt where conditions are optimal for maximal cell division and crypt orientation (7). Mutation of the $A P C$ gene causes abnormal crypt fission, disorientation of the crypts, and increased crypt production (7). Based on that knowledge, the present findings suggest that the asymmetric fission in colorectal crypts might be the result of unbalanced WNT signaling in areas adjacent to mucosal ulcers in UC.

In the three colectomies, none to occasional CCCs were found in large areas with severe chronic inflammation (Figure 1f). Importantly, other diseases of the colonic mucosa with chronic inflammation such as diverticular disease-associated colitis (9), diversion colitis (10), lymphocytic colitis (11), radiation colitis (12), collagenous colitis (13), ischemic colitis (14), and chronic colitis in Behçet's disease (15), show none to occasional CCCs. The same is valid for non-specific ulcers of the colon (16). Obviously, the development of CCCs bordering ulcers in UC is not influenced by protracted chronic mucosal inflammation or by unspecific ulcers of the colon.

Since neither chronic mucosal inflammation per se, nor unspecific ulcers of the colon are central for the formation of CCCs, it is suggested that crypt distortions of the nonulcerated colonic mucosa in patients with UC might mirror formerly healed mucosal ulcerations.

\section{References}

1 Thompson EM, Price AB, Altman DG, Sowter C and Slavin G: Quantitation in inflammatory bowel disease using computerised interactive image analysis. J Clin Pathol 38: 631-638, 1985.

2 Krease M, Stroup J, Som M and Shepard B: Fulminant ulcerative colitis complicated by treatment-refractory bacteremia. Proc (Bayl Univ Med Cent) 29: 407-408, 2016.

3 Christensen B, Hanauer SB, Erlich J, Kassim O, Gibson PR, Turner JR, Hart J and Rubin DT: Histologic Normalization occurs in ulcerative colitis and is associated with improved clinical outcomes. Clin Gastroenterol Hepatol pii: S15423565(17)30196-9, 2017.
4 Allen DC, Hamilton PW, Watt PC and Biggart JD: Architectural morphometry in ulcerative colitis with dysplasia. Histopathology 12: 611-621, 1988.

5 Rubio CA: Corrupted colonic crypt fission in carcinogen-treated rats. PLoS One 12: e0172824, 2017.

6 Rubio CA: Are corrupted non-dysplastic colonic crypts the first histological event in experimental colonic carcinogenesis? Anticancer Res 37: 2265-2268, 2017.

7 Boman BM and Fields JZ: An APC:WNT counter-current-like mechanism regulates cell division along the human colonic crypt axis: a mechanism that explains how apc mutations induce proliferative abnormalities that drive colon cancer development. Front Oncol 3: 244-250, 2013.

8 Rubio CA. al tract. Chapter 10. In: Stem Cell, Regenerative Medicine and Cancer. Singh SR (eds.). Haupauge, NY, USA, Nova Science Publ Inc., pp. 279-308. 2011.

9 Ludeman L, Warren BF and Shepherd NA: The pathology of diverticular disease. Best Pract Res Clin Gastroenterol 16: 543562, 2002.

8 Rubio CA, Ásmundsson J, Silva P, Illies C, Hartman J and Kis L: Lymphoid aggregates in Crohn's colitis and mucosal immunity. Virchows Arch 463: 637-642, 2013.

9 Rubio CA: The Natural Antimicrobial Enzyme Lysozyme is UpRegulated in Gastrointestinal Inflammatory Conditions. Pathogens 3: 73-92, 2014.

10 Nielsen OH, Vainer B and Rask-Madsen J: Non-IBD and noninfectious colitis. Nat Clin Pract Gastroenterol Hepatol 5: 2839, 2008.

11 Rubio CA, Orrego A, Höög A, Porwitz A, Petersson F, Elmberger G, Glaessgen A, Eriksson E, Kanter L, Jaremko G, Egevad L, Laforga J, Liljefors M, Löfdahl B, Norman P, Larsson O, Wanat R, Wejde J, Zickert P, Björk J, Caini S, Palli D and Nesi G: Quantitative assessment of the subepithelial collagen band does not increase the accuracy of diagnosis of collagenous colitis. Am J Clin Pathol 130: 375-381, 2008.

12 Feuerstadt $\mathrm{P}$ and Brandt LJ: Update on Colon Ischemia: Recent Insights and Advances. Curr Gastroenterol Rep 17: 45-52, 2015.

15 Lee RG: The colitis of Behçet's syndrome. Am J Surg Pathol 10: 888-893, 1986.

16 Rubio CA and Nydahl S: "Nonspecific" erosions and ulcers of the colonic mucosa. Dig Dis Sci 39: 821-826, 1994.
Received April 14, 2017

Revised April 27, 2017

Accepted April 28, 2017 